\title{
Quantification of Sofosbuvir and Velpatasvir in Human Plasma using LCMS/MS Technique -Application to Pharmacokinetic Study
}

\author{
Kishore Konam ${ }^{1, *}$, Somasekhar Reddy ${ }^{2}$ \\ 'Department of Pharmaceutical Sciences, Jawaharlal Nehru University of Technology, Ananthapuramu, Andhra Pradesh, INDIA. \\ 2Department of Pharmacology, Raghavendra Institute of Pharmaceutical Education and Research, Chiyyedu Post, Ananthapuramu, Andhra Pradesh, INDIA.
}

\begin{abstract}
Objective: An effective high-performance Liquid Chromatography-tandem Mass Spectrophotometric (LCMS/MS) method was developed for the simultaneous quantitation of sofosbuvir (SOFOS) and Velpatasvir (VELPA) in spiked human plasma. Methods: The extraction of both analytes from plasma was performed by Liquid-Liquid Extraction (LLE) technique. Separation was achieved on a Zorbax $\mathrm{C}_{18}$ Stable Bond (SB), $\mathrm{C}_{18}(4.6 \mathrm{~mm}$ id $\times 50 \mathrm{~mm})$ analytical column using acetonitrile: $1 \%$ formic acid (50:50) $\mathrm{v} / \mathrm{v}$ with a flow rate of $600 \mu \mathrm{l} / \mathrm{min}$. The MS/MS analysis was performed in Multiple Reaction Monitoring (MRM) to obtain the product ion $\mathrm{m} / \mathrm{z} 530 \rightarrow 242.3$ for SOFOS, $\mathrm{m} / \mathrm{z} 883.8 \rightarrow 643.0$ for VELPA and $\mathrm{m} / z 889.5 \rightarrow 732.6$ for internal standard (ledipasvir). Results: The calibration curve was found linear over the range of $0.5 \rightarrow 5000 \mathrm{ng} / \mathrm{ml}$ for SOFOS and $1.5 \rightarrow 2000 \mathrm{ng} / \mathrm{ml}$ for VELPA. Intra and inerday accuracy (\% nominal $98 \rightarrow 102 \%$ ), precision ( $\%$ CV $\leq 3.8 \%$ ) was excellent. Matrix effect (matrix factor 1.340 for SOFOS and 1.004 for VELPA), selectivity $(\%$ interference $=0$ ) with a extraction recovery of $96.70 \% \rightarrow 98.30 \%$. The stability (\% nominal $95.85 \rightarrow 98.90 \%$ ) of all types
\end{abstract}

were within acceptable limit. Conclusion: The proposed method was applied successfully for the pharmacokinetic study of marketed dosage form in rabbit blood samples with single oral human equivalents dose. The developed method has further applied during clinical and preclinical trials in human and other experimental animals

Key words: Sofosbuvir, Velpatasvir, Bioanalytical, LC-MS/MS, Pharmacokinetic.

Correspondence

Mr. Kishore Konam,

Department of Pharmaceutical Sciences, Jawaharlal NehruTechnological University, Ananthapuramu-510051, Andhra Pradesh, INDIA.

Phone: +91-918099879341

Email: kishorekorem19@gmail.com

DOI: 10.5530/jyp.2019.11.54

\section{INTRODUCTION}

Hepatitis $\mathrm{C}$ is a liver disorder caused by the hepatitis $\mathrm{C}$ virus. It is a blood born virus and common modes of transfer is through a small quantity of blood, can be happened through unsafe injection practices, unsafe healthcare, unsafe blood transfusion. ${ }^{1}$ Approximately 3 lacks 99 thousand people die each year. Hepatitis C occurs mostly from hepatocellular carcinoma and cirrhosis. ${ }^{2}$ Various antiviral agents are available from Hepatitis $\mathrm{C}$ but the direct acting antiviral combination medications shows promising effects on chronic hepatitis $\mathrm{C}^{3}$ sofosbuvir is a direct acting antiviral used in combinations with Velpatasvir. Sofosbuvir with molecular formula $\mathrm{C}_{22} \mathrm{H}_{29} \mathrm{FN}_{3} \mathrm{O}_{9} \mathrm{P}$, is prodrug analogue, ${ }^{4}$ which on metabolites into its active form to deoxy- $2 \infty$ fluoro, $\mathrm{BC}$ - methyl uridine- 5 triphosphate. ${ }^{5}$ The chemical structure was shown in Figure 1. Velpatasvir is also a direct acting medication used as a part of combination thereby ${ }^{6}$ it also place a key role for the inhibition of hepatitis $\mathrm{C}$ viral replications. ${ }^{7}$ Treatment option for chronic hepatitis $\mathrm{C}$ has advanced with direct acting antiviral combination since $2011 .^{8}$ Though various analytical methods like UV, HPLC, has been utilised ${ }^{9}$ but only two methods has been reported for the estimation of this combination in spiked plasma. In reported method, ${ }^{10}$ utilised three mobile phase combination and also utilised protein precipitation technique for the extraction of drug from the matrix. The disadvantage is that most of the time the matrix component is efficiently removed and may co elute with analyte and lowered the signal for the analyte. The linearity range of the reported method is 5-5000 and $10-1500 \mathrm{ng} / \mathrm{ml}$. The lower limit can be further reduce to improve the sensitivity. The average extraction recovery is $80-81 \%$, which is not satisfactory. The retention of internal standard and sofosbuvir is too close to each other. In another method ${ }^{11}$ used also protein precipitation technique which has several disadvantages that was discussed earlier, therefore the interference was found in the MRM signals. These possible disadvantages in the reported methods limits their acceptability for further clinical and other study. Therefore a sensitive, fast and easy method is spiked human plasma was planned and developed by limiting all disadvantages with the use of liquid-liquid extraction technique for the drugs from spiked plasma with better extraction recovert, analysis time and good recovery. An authentication of the developed method was proven using rabbit plasma sample. So, the present method can be better applicable for further bioequivalence, toxicity and other forensic study.

\section{MATERIALS AND METHODS}

Sofosbuvir and Velpatasvir with stated purity of $99.74 \%$ were obtained from Natco Pharmaceutical Pvt. Ltd. Hyderabad, Telangana, India. ledipasvir, purity $99.86 \%$ used as Internal Standard (IS) was kindly provided by Natco pharmaceutical Ltd., Hyderabad, India as a gift sample. Acetonitrile and formic acid was purchased from Sigma- Aldrich with $\geq$ $99.8 \%$ purity. K2 EDTA human plasma was obtained from Valley Biomedical, 121 Industrial Dr, Winchester, VA 22602, USA.

\section{HPLC operating conditions}

An isocratic elution technique considered with acetonitrile: $1 \%$ formic acid (50:50) v/v, as a mobile phase and a Zorbax $\mathrm{C}_{18}$ Stable Bond (SB), 
<smiles>CC(C)OC(=O)[C@H](C)NP(=O)(OCC1OC2C[C@H]1C(O)[C@H]2n1ccc(=O)[nH]c1=O)Oc1ccccc1</smiles>

A

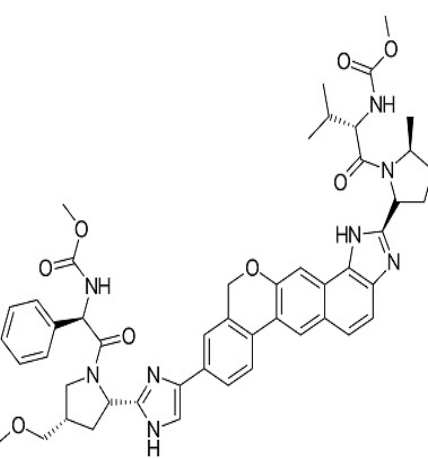

B
Figure 1: Chemical structure of Sofosbuvir (A) and Velpatasvir (B).

Table 1: Optimized mass spectrophotometric parameters for analytes and internal standard.

\begin{tabular}{|c|c|c|c|}
\hline Parameters & Sofosbuvir & Velpatasvir & $\begin{array}{l}\text { Ledipasvir } \\
\text { (Internal } \\
\text { standard) }\end{array}$ \\
\hline \multirow[t]{2}{*}{ MRM } & Parent $m / z$ & Parent $m / z$ & Parent $m / z$ \\
\hline & Daughter $m / z$ & Daughter $m / z$ & Daughter $m / z$ \\
\hline Ion spray voltage & 5400 & 5400 & 5400 \\
\hline Source temperature & 140 & 140 & 140 \\
\hline Curtain gas 1 & 41 & 41 & 41 \\
\hline Dewll time (msec) & 202 & 202 & 202 \\
\hline $\begin{array}{l}\text { Declustering potential } \\
\text { (DP) }\end{array}$ & 42 & 42 & 42 \\
\hline Capillary (kv) & 3.38 & 3.38 & 3.38 \\
\hline Cone (v) & 40 & 40 & 40 \\
\hline Collision energy (CE) & $31.5 \mathrm{ev}$ & $31.5 \mathrm{ev}$ & $31.5 \mathrm{ev}$ \\
\hline Extractor(v) & 6 & 6 & 6 \\
\hline RF lens (v) & 0 & 0 & 0 \\
\hline $\begin{array}{c}\text { Desolvation } \\
\text { temperature }\left({ }^{\circ} \mathrm{C}\right)\end{array}$ & 800 & 800 & 800 \\
\hline $\begin{array}{l}\text { Desolvation gas flow } \\
\qquad(\mathrm{L} / \mathrm{h})\end{array}$ & 800 & 800 & 800 \\
\hline
\end{tabular}

$\mathrm{C}_{18}(4.6 \mathrm{~mm}$ id x $50 \mathrm{~mm}$ ) analytical column (Agilent Technologies India Pvt. Ltd. Madhapur, Hyderabad, India). The flow rate $600 \mu \mathrm{l} / \mathrm{min}$ was set in to ESI-MS chamber. Under these condition retention time of SOFOS was $1.13 \pm 0.3 \mathrm{~min}, 1.32 \pm 0.3 \mathrm{~min}$ for VELPA and $12.5 \pm 0.3$ for internal standard. Column effluent was introduced into mass and run time was maintained up to $2 \mathrm{~min}$.

\section{Mass spectrometry operating conditions}

API-3000 triple quadrupole mass spectrometer (AB SCIEX, Foster City, $\mathrm{CA} /$ concord and Ontario, Canada) equipped with an electrospray ionisation source (ESI) mass spectrophotometer was used and operated in a positive ion mode at $800^{\circ} \mathrm{C}$ desolvation temperature. The ion source parameters for examples capillary voltages $3.38 \mathrm{kv}$, extraction cone voltage $40 \mathrm{KV}$, source temperature $140^{\circ} \mathrm{C}$, desolvation gas flow $800 \mathrm{~L} / \mathrm{h}$ and several other parameters were also fixed for the analysis of both SOFOS, VELPA and IS were summarised in Table 1. Ions were detected in multiple reaction monitoring by monitoring the transition pairs

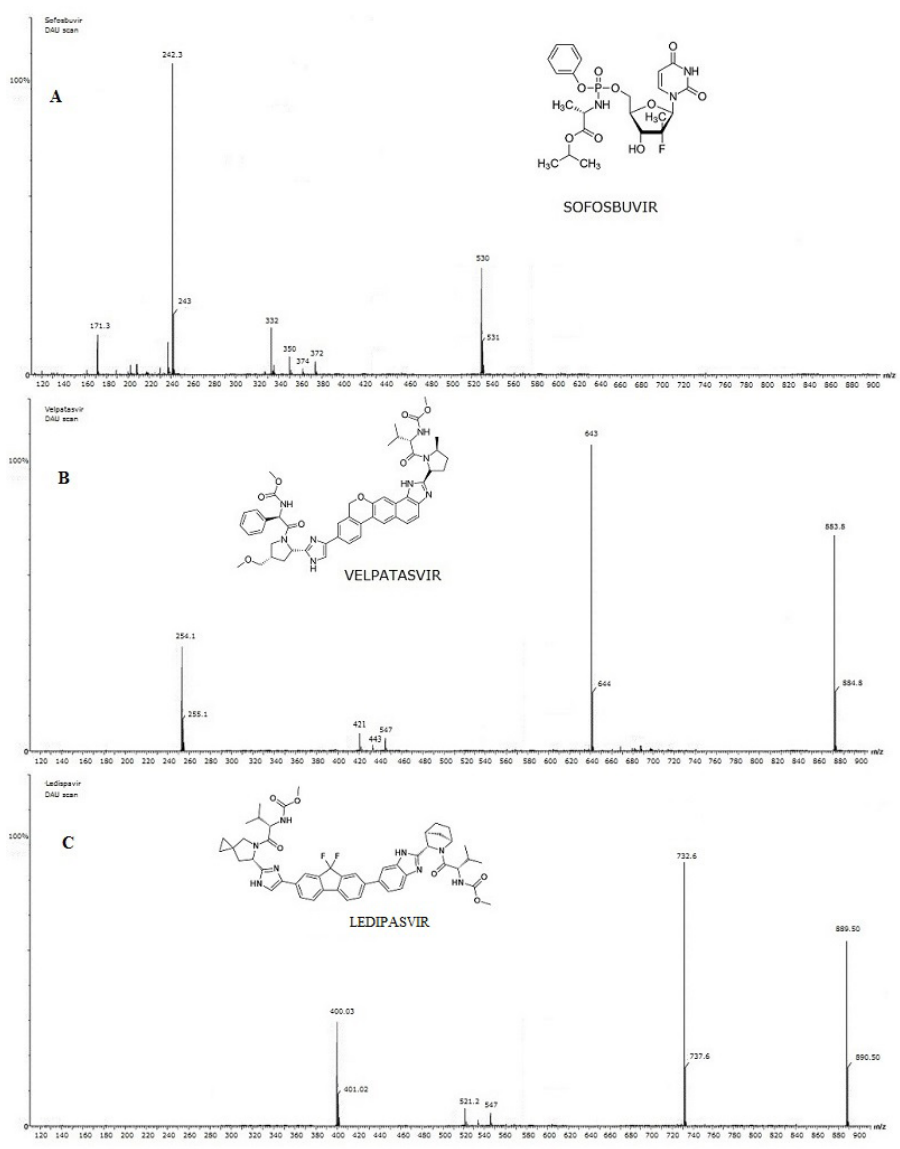

Figure 2: Product ion mass spectra $[\mathrm{M}+\mathrm{H}]^{+}$of Sofosbuvir (A), Velpatasvir (B) and Ledipasvir (C).

of $m / z 530 \rightarrow 242.3$ for SOFOS, $m / z 883.8 \rightarrow 643.0$ for VELPA and $m / z$ $889.5 \rightarrow 732.6$ for internal standard was shown in Figure 2.

\section{Preparation of calibration curve (CC) and quality control (QC) samples}

The calibration curve and quality control samples of SOFOS, VELPA and internal standard were prepared from the diluted aqueous stock solutions, which were prepared separately using $4 \mathrm{ml}$ of HPLC grade acetonitrile as a dissolving solvent for $10 \mathrm{mg}$ of SOFOS, VELPA and IS, the volume was made up to $10 \mathrm{~mL}$ to achieve the stock concentration $1 \mathrm{mg} / \mathrm{mL}$. The plasma spiked CC samples were prepared in the range of $0.5-5000 \mathrm{ng} / \mathrm{mL}$. The solutions were prepared by withdrawing $0.25 \mathrm{~mL}$ from the different aqueous calibration curve concentrations and transferred to pre labelled volumetric flasks, finally made the volume up to $5 \mathrm{ml}$ with K2-EDTA human plasma to achieve the concentrations 0.5 , 1, 5, 20, 40, 100, 200, 500, 1000, 2000, 3000, 4000 and 5000 ng/ml. For plasma spiked QC samples different concentration of SOFOS, VELPA and internal standard were pipette out from the working stock solutions (40000-100ng/ml) and transferred into pre labelled volumetric flask. The volumes were made up to $5 \mathrm{ml}$ with K2- EDTA human plasma to achieved various levels of QC samples, $1 \mathrm{ng} / \mathrm{ml}$ (LLOQ, lower limit of quantitation), $50 \mathrm{ng} / \mathrm{ml}$ (LQC, lower quality control), $500 \mathrm{ng} / \mathrm{ml}$ (MQC-1, medium quality control-1), $1000 \mathrm{ng} / \mathrm{ml}$ (MQC-2, medium quality control-2), $2000 \mathrm{ng} / \mathrm{ml}$ (HQC, high quality control), $4000 \mathrm{ng} / \mathrm{ml}$ (DIQC, dilution integrity quality control). Individual analyte stock solution was used to spike into CC and QC samples and prepared samples (5ml each) were stored inside the deep freezer at $-40^{\circ} \mathrm{C}$. 


\section{Sample preparation}

A liquid-liquid extraction technique was used for the sample preparation. The plasma samples of SOFOS, VELPA and internal standard for the quality control samples and calibration curve samples were thawed at room temperature and vortexed the plasma samples to ensure the complete mixing. 500 $\mu$ of plasma samples were withdrawn and placed in a different stopper flask. $10 \mu$ of internal standard $(100 \mathrm{ng} / \mathrm{ml})$ was added to each stoppard flask and vortexes except the blank plasma samples ( $10 \mu \mathrm{l}$ of diluent) was added. $2 \mathrm{ml}$ ethyl acetate was added as an extracting solvent to each flask and shaken for $20 \mathrm{~min}$ with reciprocating shaker at $400 \mathrm{rpm}$. Samples were then centrifuged at $3000 \mathrm{rpm}$ for $10 \mathrm{~min}$ at $25^{\circ} \mathrm{C}$. The supernatant layer was transferred into pre labelled tubes and evaporated to dryness under nitrogen gas at $40^{\circ} \mathrm{C}$. Samples were reconstituted with $500 \mu \mathrm{l}$ of mobile phase and transferred into autosampler. $10 \mu \mathrm{l}$ of each sample were injected into LC-MS/MS system.

\section{Method Validation}

The developed method was validated as per the guidelines of bioanalytical method development and validation of by USFDA. ${ }^{12}$ The developed method was validated for matrix effect, carry over effect, recovery, sensitivity, accuracy, precision and different stability parameters.

\section{Carry over effect}

It was performed to investigate the effect of carrying analytes and internal standard in different sections of the system. Six replicates of the extracted blank matrix and six replicates of the extracted high concentration of analytes at the calibration curve range (ULOQ), six extracted LLOQ concentrations of the analytes and internal standard were prepared and injected as per sequence i.e. first six injections were extracted LLOQ samples, extracted ULOQ samples followed by blank plasma. ULOQ and blank plasma samples were injected alternatively.

\section{Matrix effect}

It was performed at two different concentration level (LQC and HQC) in eight replicates for both analytes and internal standard. One hemolytic and one lipemic plasma lots have been selected for the study. One set of each sample were spiked with blank matrices (hemolytic), another set of each the sample (LQC and HQC) with internal standard was spiked with lipemic plasma. Six replicates of aqueous samples equivalent to final LQC and HQC concentrations were prepared by spiking the analytes (SOFOS and VELPA) with internal standard (LEDISPA) to reconstituted solution and injected individually. IS normalized matrix factor was calculated and the variability in IS-normalized matrix factor was measured by the coefficient of variation and it should be should be less than $15 \%$.

\section{Matrix selectivity and specificity}

It was evaluated by analysing the plasma of six different lots, including one haemolytic and lipemic plasma to investigate the interference the retention time of the analytes and internal standard. The interference at the retention times of the drugs by comparing the response in the blank plasma, against the response of LLOQ was evaluated. The interference at the retention time of internal standard also evaluated against the response of the extracted internal standard in LLOQ sample.

\section{Analytes selectivity}

Analytes selectivity study was performed to investigate the internal standards interference at analyte's retention time. Six replicates of matrix blank with internal standard was injected, if any area for analytes was found was compared with mean area of the analytes obtained with LLOQ concentration injected. Similarly internal standards selectivity was also investigated for this six replicates of matrix blank with drugs was in injected at ULOQ level and area of internal standard if obtained, compared with mean area of internal standard at LLOQ level.

\section{Precision and accuracy}

The intra and interday precision and accuracy study of the developed method was conducted using several QC samples at the level of LLOQ, LQC, MQC and HQC in six replicates and the concentrations in these level was calculated followed by standard deviation, $\% \mathrm{CV}$ for precision and $\%$ nominal for accuracy for each replicates.

\section{Linearity}

The developed method was assessed for linearity in the concentration range of $0.5-5000 \mathrm{ng} / \mathrm{ml}$, for SOFOS and $1.5-2000 \mathrm{ng} / \mathrm{ml}$ for VELPA. Several CC (calibration curve) samples has been prepared by spiking with human plasma and processed. The calibration curve was constructed by using a regression equation with a weighing factor $1 /$ (concentration ratio $)^{2}$ of the drug to internal standard concentration to produce the best fit for the concentration/response relationship. The acceptance criteria for the linearity is that the $r^{2}$ (coefficient of correlation) should be $\geq 0.98$ and for LLOQ concentration $\pm 20 \%$ deviation can be acceptable from nominal value but $\pm 15 \%$ deviation should maintain other than LLOQ concentration.

\section{Dilution integrity}

For the dilution integrity 12 sets of QC stock solution were prepared by spiking 1.5 times of the highest standard concentration. Six sets of dilution integrity samples were prepared by diluting 2 times and another six samples by 4 times dilution were made. These samples were analysed and concentration were calculated multiplying suitable dilution factors, 2 (for two times dilution) and 4 (for four times dilution).

\section{Recovery study}

This study was performed to evaluate the extraction efficiency of a analytical process, by comparing the peak response from extracted and non-extracted samples. Six LQC, MQC and HQC samples has been prepared freshly and these samples were processed by adding internal standard and injected. Eighteen blank matrix samples were spiked with six sets of each LQC, MQC and HQC with internal standard for nonextracted samples and injected. Six non extracted samples of each 3 levels were prepared by spiking $10 \mu \mathrm{l}$ of analytes and $10 \mu \mathrm{l}$ of internal standard in extracted blank plasma. Overall mean \% recovery was calculated.

\section{Ruggedness}

For the study of the developed method, one precision and accuracy batch of samples were processed and analysed with different columns of same make and with different reagent lots.

\section{Stability Studies}

In the Bench top, wet extract, freeze thaw, autosampler, short term and long-term stability study of the developed method, freshly prepared calibration curve samples and quality control samples were prepared and analysed at low middle, high level. Concentration response linearity data was collected and used to calculate the concentration of stability samples.

\section{Room temperature stability study}

It was conducted using the prepared stock solution for a period of $6 \mathrm{hr}$. Fresh stock solution of both analytes and internal standard were prepared. The final dilution of both stock solution (stability samples) and fresh stock solution (comparison sample) was done, which is equivalent to final middle quality control analytes and internal standard. Six replicates of fresh and comparison samples were injected immediately and \% of stability was calculated. 


\section{Bench top stability}

For the bench top stability study six sets of LQC and HQC samples were collected from the deep freezer and placed unprocessed for a period of $12 \mathrm{hr}$. After that period six sets of fresh quality control samples (low, middle, high) and calibration samples were prepared. Bench top stability samples were processed, analysed along with fresh samples. Concentration was calculated from the linearity data.

\section{Refrigerator stock solution stability}

For this type of stability study six replicates of stock solution was prepared and stored at refrigerator at $2-8^{\circ} \mathrm{C}$ for 4 days. On the day of study fresh standard stock solution was prepared comparison sample similar to final MQC concentration of the analytes with final concentration of internal standard in reconstituted solution. All stability and comparison samples were injected immediately. Percentage of stability was also calculated for both the analytes and internal standard

\section{Autosampler stability}

For this stability study six sets of QC samples were prepared in LQC and HQC level and kept in autosampler for 3 days. All the stability samples were quantified against the freshly prepared spiked calibration curve and quality control samples at low middle and high level.

\section{Freeze thaw stability}

For this study four freeze thaw cycles was conducted. Six replicates of LQC and HQC samples were prepared and stored in deep freezer at $-70^{\circ} \mathrm{C}$. After $24 \mathrm{hr}$ of freezing, first six samples were withdrawn and thawed at room temperature and refreeze again. Similar way remaining samples were also withdrawn after next $12 \mathrm{hr}$ followed by another $12 \mathrm{hr}$ and refreeze again. Entire range of samples were processed after suitable 4 cycles. Stability samples were analyzed along with freshly spiked calibration samples and quality control samples at low mid and high level.

\section{Wet extract stability}

For this study six LQC and HQC samples of six replicates were prepared, processed and kept $24 \mathrm{hr}$ at room temperature $\left(20 \pm 5^{\circ} \mathrm{C}\right)$. After the stability period the samples were injected with freshly prepared spiked calibration curve samples and quality control samples at low middle and high level. The amount of analytes in stability samples was calculated in compared with freshly prepared samples.

\section{Short term stability at $-20^{\circ} \mathrm{C}$}

Quality control samples at low and high level were prepared for six sets and stored at deep freezer at $-20^{\circ} \mathrm{C}$ after spiking for 3 days. On the day of study samples were processed along with freshly prepared quality control samples in all levels and calibration curve samples. The concentration of the stability samples were calculated in comparison to freshly prepared samples.

\section{Long term stability at $-70^{\circ} \mathrm{C}$}

This was investigated with LQC and HQC samples kept for 30 days at $-70^{\circ} \mathrm{C}$. On the day evaluation six sets of long-term quality control samples (LQC and HQC) were withdrawn and processed them with freshly prepared calibration curve and quality control samples. Calibration curve data was utilised to quantify all the samples.

\section{Pharmacokinetic study}

This study was performed using NZ white rabbit $(n=3,2.5 \mathrm{~kg}$ each, 2 male, male: female 2:1) model using the permission of IAEC permission, no. 1604/PO/Re/S/13/CPCSEA. To assess the applicability of the developed method, it was carried at oral dose of $1.35 \mathrm{mg}$ of SOFOS and for VELPA is $5.3 \mathrm{mg}$ for Etizolam. The orally administered dose was human equivalent dose for this particular combination of marketed formulation
"Velamen" (sofosbuvir $400 \mathrm{mg}$ and velpatasvir 100mg). The dose was calculated as per the US-FDA guidelines for the calculation of equivalent dose. ${ }^{13,14}$ Silicon gastric intubation tube was utilised for the administration of dosage form to their respective group. Blood samples $(1 \mathrm{ml})$ was collected from the marginal ear vein into polypropylene tube (K2 EDTA, J.K diagnostic- Rajkot) at $0,0.5,1,1.5,2,2.5,3,3.5,5,8,10 \mathrm{~h}$ post dose. Plasma was separated immediately by centrifugation (2000rpm, $7 \mathrm{~min}$ ) and stored at $-80^{\circ} \mathrm{C}$ until analysis. The plasma samples were spiked with internal standard and processed as per sample preparation technique described earlier. The plasma concentration and time data were analysed with a compartment model, using PK solver. 2 a Manu driven adding programme for MS excel (18).

\section{One batch intraday accuracy and precision study of rabbit blood samples}

This was performed to strengthen the applicability of the developed method. The blood samples that were collected for the pharmacokinetic study from the rabbits were also subjected to one batch intraday accuracy and precision study. The collected blood samples were further processed as per the sample preparation method with internal standard, at LLOQ, LQC, MQC-1, MQC-II and HQC level.

\section{RESULTS}

\section{Mass spectrometry}

Both protonated analyses and internal standard $[\mathrm{M}+\mathrm{H}]^{+}$ion was the parent ion in the quadropole- 1 segment and used as precursor ion to obtained product ion spectra in quadropole-3. All the optimized mass parameters has been cited in Table 1 and product ion mass spectra was shown in Figure 2.

\section{Method development}

Several combination of buffers and acetonitrile has been utilized in initial trials, different types of columns like C-8, $\mathrm{C}_{18}$ of Waters symmetry shield, Zorbax, hypersil, kromasil has been used and finally, Zorbax $\mathrm{C}_{18}$ Stable Bond (SB) analytical column with the mobile phase composed of acetonitrile and $1 \%$ formic acid in the volume ratio (50:50) v/v has selected. Ledipasvir was selected as an internal standard. Under this optimized condition the retention time of SOFOS was $1.13 \pm 0.3 \mathrm{~min}, 1.32 \pm 0.3 \mathrm{~min}$ for VELPA and 12.5 \pm 0.3 for internal standard, shown as MRM chromatograms at LQC and HQC level in Figure 3 and 4.

\section{Prevalidation and validation}

In carry over test the response for blank samples was found 0 , therefore the calculated \% carry over is 0 . "Internal standard normalized factor" was calculated to determine the matrix effect on the analytes. The $\% \mathrm{CV}$ of Is normalized factor was found 5.32 and 7.81 for SOFOS LQC and HQC samples, whereas 6.53 and 5.03 for VELPA LQC and HQC samples. \% CV of between batch precision for LLOQ, LQC and MQC-I, MQC-II and HQC samples of SOFOS was found 2.13, 1.03, 1.18, 1.23 and 1.68. Similarly, for VELPA it was $0.63,2.19,0.32,0.06$ and 1.23 . Between the batch accuracy for LLOQ, LQC and MQC-I, MQC-II and HQC were found $95.83,96.23,98.03,97.53$ and $98.71 \%$ for SOFOS and for VELPA accuracy values were $95.3,97.18,98.63,98.33$ and $98.90 \%$, details were shown in Table 2. In matrix selectivity study the response of interfering peaks at the retention time of both analyses and is was found $0 \%$ of the mean drug response. A regression equation of $1 /$ (concentration ratio) ${ }^{2}$ of drugs to internal standard concentration was found 0.99 in the concentration range of $0.5-4000 \mathrm{ng} / \mathrm{mL}$ for SOFOS and $1.5-200 \mathrm{ng} / \mathrm{mL}$ for VELPA. In dilution integrity study (2 and 4 times) at ULOQ level, the precision and accuracy of SOFOS for dilution factor 2 were found 0.085 and $98.96 \%$. For VELPA the values were 0.147 and $98.96 \%$. Similarly, 

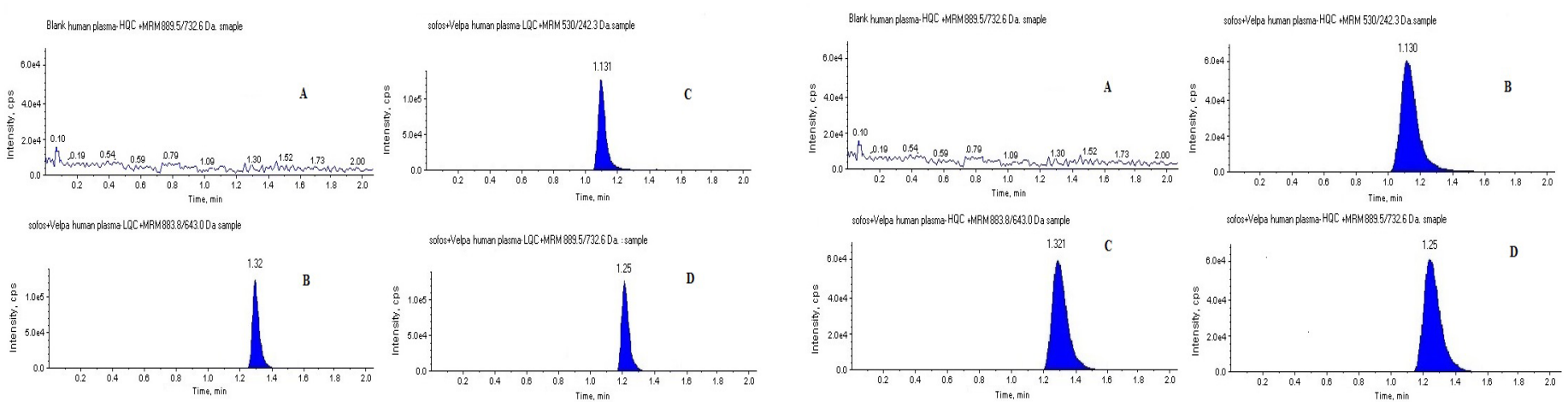

Figure 3: MRM chromatogram of Blank (A), Sofosbuvir (B), Velpatasvir (C) and Ledipasvir (D) At LQC level.

Figure 4: MRM chromatogram of Blank (A), Sofosbuvir (B), Velpatasvir (C) and Ledipasvir (D) At HQC level.

Table 2: Intraday and Interday accuracy and precision of determination of Sofosbuvir and Velpatasvir in human plasma.

\begin{tabular}{|c|c|c|c|c|c|c|c|c|c|}
\hline \multirow[t]{2}{*}{ QC } & \multirow{2}{*}{$\begin{array}{c}\begin{array}{c}\text { Sofosbuvir measured } \\
\text { concentration (ng/ml) }\end{array} \\
\text { Run* }\end{array}$} & \multirow[b]{2}{*}{ Mean } & \multirow[b]{2}{*}{ SD } & \multirow[b]{2}{*}{$\%$ CV } & \multirow[b]{2}{*}{$\%$ Nominal } & \multicolumn{4}{|c|}{ Velpatasvir measured concentration (ng/ml) } \\
\hline & & & & & & Mean & SD & $\% C V$ & \%Nominal \\
\hline \multirow{4}{*}{$\begin{array}{c}\text { LLOQ } \\
(1 \mathrm{ng} / \mathrm{mL})\end{array}$} & 1 & 0.96 & 0.19 & 3.18 & 96.02 & 0.95 & 0.87 & 2.05 & 95.20 \\
\hline & 2 & 0.98 & 0.36 & 0.08 & 98.5 & 0.93 & 0.76 & 1.56 & 93.65 \\
\hline & 3 & 0.89 & 0.88 & 0.19 & 89.60 & 0.97 & 0.03 & 2.98 & 97.08 \\
\hline & 4 & 0.91 & 0.75 & 0.33 & 91.05 & 0.92 & 0.06 & 1.75 & 93.52 \\
\hline \multirow{4}{*}{$\begin{array}{c}\text { LQC } \\
(50 \mathrm{ng} / \mathrm{mL})\end{array}$} & 1 & 48.76 & 0.35 & 0.69 & 97.03 & 49.05 & 0.11 & 0.34 & 98.12 \\
\hline & 2 & 48.97 & 0.89 & 1.75 & 97.08 & 49.19 & 0.52 & 3.50 & 98.32 \\
\hline & 3 & 49.08 & 0.12 & 0.79 & 98.40 & 48.76 & 0.06 & 0.41 & 97.51 \\
\hline & 4 & 49.71 & 0.04 & 0.66 & 99.42 & 48.43 & 0.06 & 0.63 & 98.86 \\
\hline \multirow{4}{*}{$\begin{array}{c}\text { MQC-1 } \\
(500 \mathrm{ng} / \mathrm{mL})\end{array}$} & 1 & 480.35 & 0.31 & 1.32 & 97.87 & 487.51 & 0.48 & 0.32 & 97.50 \\
\hline & 2 & 491.30 & 0.35 & 1.03 & 98.26 & 492.51 & 0.07 & 0.35 & 98.50 \\
\hline & 3 & 492.47 & 0.53 & 1.01 & 98.49 & 493.53 & 0.39 & 0.26 & 98.70 \\
\hline & 4 & 489.75 & 0.89 & 0.92 & 97.95 & 491.86 & 0.06 & 0.04 & 98.37 \\
\hline \multirow{4}{*}{$\begin{array}{c}\text { MQC-II } \\
(1000 \mathrm{ng} / \mathrm{mL})\end{array}$} & 1 & 969.13 & 0.85 & 1.13 & 96.91 & 988.18 & 1.19 & 0.07 & 98.81 \\
\hline & 2 & 983.86 & 0.73 & 1.20 & 98.38 & 970.20 & 0.50 & 0.03 & 97.07 \\
\hline & 3 & 989.5 & 1.19 & 1.23 & 98.95 & 978.10 & 1.16 & 0.07 & 97.81 \\
\hline & 4 & 989.20 & 1.21 & 1.03 & 98.12 & 980.30 & 1.32 & 0.08 & 98.03 \\
\hline \multirow{5}{*}{$\begin{array}{c}\text { HQC } \\
(2000 \mathrm{ng} / \mathrm{mL})\end{array}$} & 1 & 1982.21 & 1.23 & 1.32 & 99.11 & 1969.30 & 0.08 & 1.01 & 98.46 \\
\hline & 2 & 1962.51 & 1.03 & 1.87 & 98.12 & 1972.59 & 0.39 & 1.23 & 98.62 \\
\hline & 3 & 1953.81 & 1.08 & 1.65 & 97.69 & 1952.63 & 0.19 & 1.29 & 97.63 \\
\hline & 4 & 1972.72 & 1.21 & 1.03 & 98.63 & 1988.49 & 0.11 & 1.31 & 99.42 \\
\hline & & & & & & \multicolumn{4}{|c|}{$\begin{array}{c}\text { Interday variations (each concentration include } 20 \\
\text { replicates) }\end{array}$} \\
\hline LLOQ & & 0.97 & 0.18 & 0.58 & 97.0 & 0.97 & 0.13 & 0.59 & 97.0 \\
\hline LQC & & 48.93 & 0.32 & 0.83 & 96.36 & 47.93 & 0.58 & 0.72 & 95.86 \\
\hline MQC-1 & & 489.31 & 0.51 & 1.32 & 97.86 & 488.13 & 0.44 & 1.38 & 97.22 \\
\hline MQC-II & & 967.76 & 0.56 & 1.53 & 98.46 & 945.57 & 0.78 & 0.95 & 97.45 \\
\hline HQC & & 1972.48 & 0.03 & 1.82 & 97.24 & 1975.31 & 0.39 & 0.87 & 97.53 \\
\hline
\end{tabular}

${ }^{\star}$ Each run includes six replicate.

for a dilution factor 4 the precision and accuracy of SOFOS values were 0.465 and 156.17 and for VELPA values are 0.183 and 101.06. The mean overall recovery of SOFOS was found $94.04 \%$ with a precision range of $2.11 \%$ to $4.90 \%$ and a percentage difference 5.31 . Similarly, overall recovery of SOFOS was found $98.80 \%$ with a precision range of $0.929 \%$ to $6.69 \%$, with a percentage difference of 14.48 . Internal standard mean overall recovery was found $98.16 \%$ which satisfied acceptance criteria. In ruggedness study within batch precision of LLOQ, LQC, MQC-I, 
Table 3: Stability data of sofosbuvir and velpatasvir.

\begin{tabular}{|c|c|c|c|c|c|c|c|c|c|}
\hline \multirow[t]{2}{*}{ QC concent-ration } & \multirow{2}{*}{$\begin{array}{c}\text { Stability } \\
\text { Type }\end{array}$} & \multicolumn{4}{|c|}{ Sofosbuvir } & \multicolumn{4}{|c|}{ Velpatasvir } \\
\hline & & Mean* & SD & $\begin{array}{c}\% \text { CV } \\
\text { (Precision) }\end{array}$ & $\begin{array}{l}\text { \% Nominal } \\
\text { (Accuracy) }\end{array}$ & Mean & SD & $\begin{array}{c}\% \text { CV } \\
\text { (Precision) }\end{array}$ & $\begin{array}{l}\text { \% Nominal } \\
\text { (Accuracy) }\end{array}$ \\
\hline \multirow{6}{*}{ LQC } & Bench top & 48.23 & 0.02 & 1.62 & 96.46 & 47.39 & 0.05 & 1.38 & 94.78 \\
\hline & Freeze thaw & 49.17 & 0.19 & 1.41 & 98.34 & 48.15 & 0.29 & 1.32 & 96.30 \\
\hline & Autosampler & 48.73 & 0.36 & 1.35 & 97.46 & 48.78 & 0.58 & 1.45 & 97.56 \\
\hline & Wet extract & 48.55 & 0.08 & 1.67 & 97.1 & 47.13 & 0.98 & 1.76 & 94.26 \\
\hline & Short term & 49.01 & 0.59 & 1.21 & 98.02 & 48.35 & 0.76 & 1.67 & 96.70 \\
\hline & Long term & 47.92 & 1.01 & 1.37 & 95.84 & 47.37 & 1.06 & 1.80 & 94.74 \\
\hline \multirow{6}{*}{ HQC } & Bench top & 1972.2 & 1.08 & 1.55 & 98.61 & 1968.13 & 1.09 & 1.54 & 99.40 \\
\hline & Freeze thaw & 1968.25 & 0.96 & 1.76 & 98.43 & 1959.73 & 0.99 & 1.85 & 97.98 \\
\hline & Autosampler & 1978.01 & 0.92 & 1.88 & 98.90 & 1981.32 & 0.97 & 1.62 & 99.06 \\
\hline & Wet extract & 1972.69 & 0.67 & 1.26 & 98.63 & 1962.59 & 0.58 & 1.88 & 98.12 \\
\hline & Short term & 1961.54 & 0.29 & 1.10 & 98.15 & 1968.11 & 0.78 & 1.92 & 98.40 \\
\hline & Long term & 1968.11 & 0.89 & 1.57 & 98.40 & 1072.18 & 0.67 & 1.58 & 98.60 \\
\hline
\end{tabular}

${ }^{*}$ Mean of six replicates

Table 4: Pharmacokinetic parameters of SOFOS and VELPA in rabbit plasma.

\begin{tabular}{ccc}
\hline $\begin{array}{c}\text { Pharmacokinetic } \\
\text { parameters }\end{array}$ & Sofosbuvir & Velpatasvir \\
\hline $\mathrm{C}_{\max }$ & 839.59 & 1013.03 \\
$\mathrm{t}_{\max }$ & 2.38 & 3.12 \\
$\mathrm{AUC}_{0-\infty}\left(\mathrm{h}^{*} \mathrm{ng} / \mathrm{ml}\right)$ & 3432.18 & 5018.2 \\
$\mathrm{AUC}_{0-\mathrm{t}}\left(\mathrm{h}^{*} \mathrm{ng} / \mathrm{ml}\right)$ & 3328.72 & 4388.8 \\
$\mathrm{t}_{1 / 2}(\mathrm{hr})$ & 1.27 & 4.32 \\
\hline
\end{tabular}

MQC-II and HQC ruggedness SOFOS samples were found 2.34\%, $1.807 \%, 0.533 \%$ and $0.545 \%$. Similarly, for VELPA ruggedness samples values were $5.208 \%, 0.563 \%, 0.410 \%$ and $1.37 \%$. The accuracy values for SOFOS were $97.53 \%, 98.60 \%, 98.30 \%, 99.12 \%$ and for VELPA values were $98.00 \%, 98.86 \%, 98.34 \%$ and $99.16 \%$.

\section{Stability studies}

The calculated \% stability for SOFOS, VELPA and internal standard were $97.4,98.4$ and 97.8 . for room temperature stability study. In refrigerator stock solution stability study at $2-8^{\circ} \mathrm{C}$ for 4 days, the calculated $\%$ of stability for SOFOS was found 101.03, for VELPA it was 98.37 and for internal standard 98.45. In bench top stability, the mean \% of nominal of LQC and HQC samples were found $96.46 \%$ and $98.61 \%$ for SOFOS. Whereas\% nominal of VELPA was found 94.78 and 99.40 in LQC and HQC. After $72 \mathrm{~h}$ of suitable stability period in autosampler, the $\%$ nominal (accuracy) was found $97.46 \%$ and $98.00 \%$ for SOFOS at LQC and HQC level, whereas \% nominal (accuracy) values for VELPA were $97.56 \%$ and $99.90 \%$ at LQC and HQC level. The four cycles of freeze thaw stability sample shows \% nominal $98.34 \%$ and $98.43 \%$ in LQC and HQC samples of SOFOS, VELPA stability samples shown $96.30 \%$ and 97.98\% in LQC and HQC level respectively. In wet extract stability study, the $\%$ nominal value for SOFOS was found $97.1 \%$ and $98.63 \%$ in LQC and HQC and for SOFOS it was $94.26 \%$ and $98.12 \%$ in both levels, satisfied acceptance criteria for stability study. In short term stability study, the calculated \% nominal for SOFOS were $98.02 \%$ and $98.15 \%$ at LQC and HQC level and for VELPA was $96.70 \%$ at LQC and $98.46 \%$ at HQC level. The mean \% nominal for long term stability were $95.85 \%$ for
LQC and $98.40 \%$ for HQC stability samples of SOFOS. Similarly the \% of nominal for VELPA were $94.74 \%$ and $98.60 \%$ at LQC and HQC stability samples. The summary of all stability study results were demonstrated in Table 3. In bench top, wet extract, freeze thaw, autosampler, short and long term $\left(-70^{\circ} \mathrm{C}, 30\right.$ days $)$ stability studies.

\section{In vivo pharmacokinetic study}

The results of pharmacokinetic parameters obtained from the study using non compartmental model, were area under the curve $\left(\mathrm{AUC}_{0}-\infty\right)$ for SOFOS $=3432.18 \pm 1092.67 \mathrm{hr} . \mathrm{ng} / \mathrm{ml}$ and for VELPA $=5018.23 \pm 109.19$ hr.ng/ml. Elimination half-life $\left(\mathrm{t}^{1 / 2}\right)$ for SOFOS $=1.26 \pm 1.03 \mathrm{~h}$ and $\mathrm{VELPA}=4.32 \pm 1.08 \mathrm{~h}$. All the other parameters were demonstrated in Table 4

In one batch accuracy and precision study of rabbit blood samples, the $\% \mathrm{CV}$ (precision) were found less than 2 and \% N(Accuracy) were found within $95-101 \%$ in all the study levels of both the SOFOS and VELPA. The details of the result shown in Table 5.

\section{DISCISSION}

The mass parameters has been optimized only after the proper tuning of mass spectrophotometer in both positive and negative ion mode for both the analytes and internal standard, finally positive ion mode with multiple reaction monitoring mode has been selected for better specificity. Several trials were conducted to optimize the chromatographic condition required for the separation of components using different mobile phases, columns.

The obtained chromatographic condition satisfactory peak shape was obtained with reasonable retention times for both analytes and internal standard. Initially several compounds were investigated to select a suitable internal standard, finally ledipasvir was selected because the retention time and other values were very reproducible and selective and lack of interference with other analytes.

The carryover test result shows that there was no interference was found at the retention time of the analytes and internal standard at ULOQ and LLOQ level. No significant effect of matrix was found in eight batch includes haemolytic and lipemic plasma. Within batch accuracy and precision results for both SOFOS and VELPA for all quality control levels were found within acceptance criteria. Therefore, the method was found 
Table 5: Interday accuracy and precision data of original rabbit plasma sample.

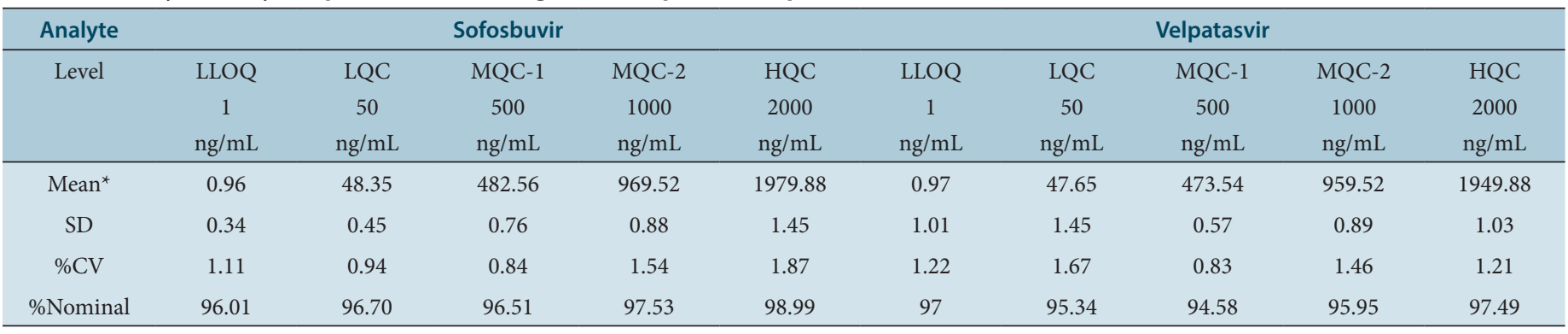

*average of six individual run.

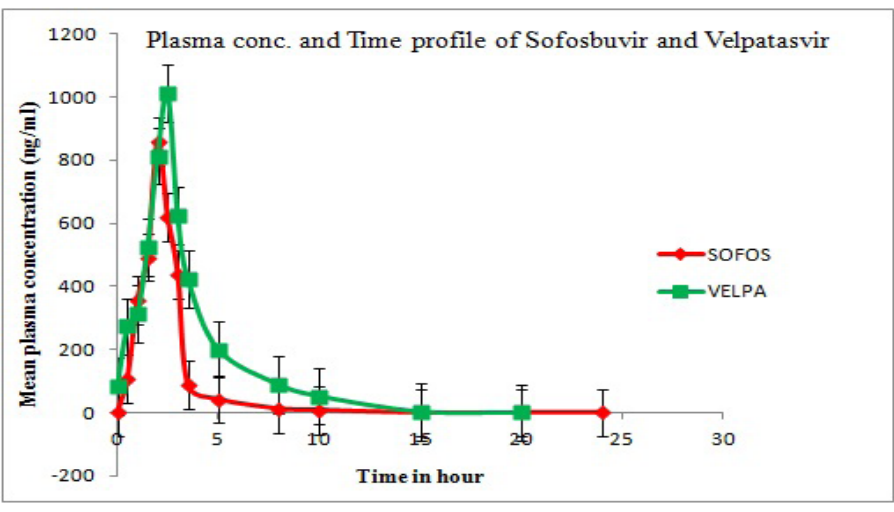

Figure 5: Mean plasma concentration ( $\mathrm{ng} / \mathrm{ml}$ ) - time (h) profile of Sofosbuvir and Velpatasvir after oral administration of marketed dosage form.

accurate and precise. Analyte selectivity study results shows that there was no interference at the retention time of both analytes in presence of internal standard and found $0 \%$ interference at the retention time of internal standard in the presence of both analytes. In the linearity study the regression equation observed best fit relationship with a correlation coefficient $\left(r^{2}\right)$. The dilution integrity study results confirm the integrity of the developed method up to 4 times of its diluted samples. The developed method was also found rugged as all the empirical evidences were within acceptance limit.

The room temperature $\left(20 \pm 5^{\circ} \mathrm{C}\right)$ stability study results was found within acceptance criteria. And the results of refrigerator stock solution stability study at $2-8^{\circ} \mathrm{C}$ for 4 days, indicates the stability of the developed method. The \% nominal of both HQC and LQC levels indicted the bench top stability of the developed method. Results of other stability samples were compared with freshly prepared samples and concentration result was back calculated from the calibration curve sample. In all types of stability study, the mean $\%$ of nominal values were found within $85 \%-115 \%$ and $\%$ CV values were less than $15 \%$, which strongly demonstrated about the stability of all quality control samples as well as stability of the developed method. In pharmacokinetic study on rabbit models the plasma concentration and time curves for both the drugs were found smooth to derive all parameters (Figure 5). The elimination half-life and clearance were well fitted with one compartmental model. The sensitivity, selectivity and specificity of the developed method was sufficient enough for the simultaneous characterization and the study of Pharmacokinetic profile of SOFOS and VELPA in rabbit model. The results of one accuracy and precision study on rabbit blood samples were within the acceptance criteria. Therefore, this study further strengthened the applicability of the developed method

\section{CONCLUSION}

The developed method is the first reporting bioanalytical method for the simultaneous estimation of sofosbuvir and velpatasvir in spiked human plasma using LC-MS/MS. The developed method was found simple, fast and convenient. The obtained pharmacokinetic parameters confirm the specific applicability of the developed method. Therefore, the present method can undoubtedly applicable for the simultaneous quantitative analysis of sofosbuvir and velpatasvir during clinical trials and toxicological study in human and other experimental animals.

\section{ACKNOWLEDGEMENT}

Authors are thankful to the management of Raghavendra Institute of Pharmaceutical Education and Research, Ananthapuramu, AP, for providing the necessary facilities to carryout the research work.

\section{CONFLICT OF INTREST}

The authors declare no conflict of interest.

\section{ABBREVIATIONS}

SOFOS: Sofosbuvir; VEPLA: Velpatasvir; LEDISPA: Ledipasvir; LCMS: Liquid chromatography and mass spectroscopy; LQC: Lower quality control; MQC: medium quality control; HQC: Highest quality control; ULOQ: Upper limit of quantitation; DIQC: dilution integrity quality control; \% CV: Percentage coefficient of variation; AUC: Area under the curve; SD: Standard deviation; CC: Calibration curve.

\section{REFERENCES}

1. Lawitz E, Mangia A, Wyles D, Hassanein T, Gordon SC, Schultz M, et al. Sofosbuvir for previously untreated chronic hepatitis C infection. N Engl J Med. 2013;69(7):678-9.

2. Kowdley KV, Lawitz E, Crespo I, Hassanein T, Davis MN, DeMicco M, et al. Sofosbuvir with pegylated interferon alfa-2a and ribavirin for treatment-naive patients with hepatitis C genotype-1 infection (ATOMIC): An open-label, randomised, multicentre phase 2 trial. Lancet. 2013;381(9883):2100-7.

3. Jacobson IM, Gordon SC, Kowdley KV, Yoshida EM, Rodriguez-Torres M, Sulkowski MS, et al. Sofosbuvir for hepatitis C genotype 2 or 3 in patients without treatment options. N Engl J Med. 2013;368(20):1867-77.

4. Zeng QL, Zhang JY, Zhang Z, Wang LF, Wang FS. Sofosbuvir and ABT-450: Terminator of hepatitis C virus. World J Gastroenterol. 2013;19(21):3199-206.

5. Herbst DA, Reddy KR. Sofosbuvir, a nucleotide polymerase inhibitor, for the treatment of chronic hepatitis C virus infection. Expert Opin Investig Drugs. 2013;22(4):527-36.

6. Gentile I, Borgia F, Buonomo AR, Castaldo G, Borgia G. A novel promising therapeutic option against hepatitis C virus: An oral nucleotide NS5B polymerase inhibitor sofosbuvir. Curr Med Chem. 2013;20(30):3733-42.

7. Soriano V, Vispo E, Mendoza C, Labarga P, Fernandez-Montero JV, Poveda E, et al. Hepatitis C therapy with HCV NS5B polymerase inhibitors. Expert Opin Pharmacother. 2013;14(9):1161-70.

8. Bhatia HK, Singh H, Gerwal N, Natt NK. Sofosbuvir: A Novel Treatment Option 
for Chronic Hepatitis C Infection. J Pharmacol Pharmacother. 2014;(4):278-84.

9. Kish T, Aziz A, Sorio M. Hepatitis C in a New Era: A Review of Current Therapies. Pharm Ther. 2017;42(5):316-29.

10. Ehab FE, Ahmed AA. Rapid bioanalytical LC-MS/MS method for the simultaneous determination of Sofosbuvir and Velpatasvir in human plasma-application to a pharmacokinetic study in Egyptian volunteers. J Chromatogr B. 2018;1102:116-24.

11. Mamdouh RR, Emad BB, Kamal AB. Novel determination of Sofosbuvir and Velpatasvir in human plasma by UPLC-MS/MS method: Application to a bioequivalence study. Biomed Chromatogr. 2018;32(11):43-7.
12. US Food and Drug Administration, Guidance for Industry: Bioanalytical Method Validation. 2013. Available:http://www.fda.gov/downloads/Drugs/GuidanceComplianceRegulatoryInformation/Guidances/UCM368107.pdf.

13. Human equivalent dose calculation. http://www.fda.gov/downloads/Drugs/.../ Guidances/UCM078932.pdf.

14. Mondal P, Sobharani S, Ramakrishna R. A Novel Simultaneous Quantification Method for Escitalopram and Etizolam in Human Plasma Using Liquid Chromatography-ESI-Tandem Mass Spectrophotometry-Application to Pharmacokinetic Study. Curr Pharm Anal. 2017;13(3):279.

Article History: Submission Date : 28-03-2019; Revised Date : 28-05-2019; Acceptance Date : 06-06-2019.

Cite this article: Konam K, Reddy S. Quantification of Sofosbuvir and Velpatasvir in Human Plasma using LCMS/MS Technique -Application to Pharmacokinetic Study. J Young Pharm. 2019;11(3):266-73. 